\title{
La construcción de pueblo en el populismo. Análisis al discurso de un presidente en Colombia (2002 - 2010)*
}

\author{
Javier A. Molina' y Laura C. Blandón ${ }^{2}$ \\ Grupo de Gestión y Política Pública, Universidad del Valle (Colombia)
}

Recibido: mayo 17 de 2015. Revisado: mayo 27 de 2016. Aceptado: octubre 31 de 2016

Referencia formato APA: Molina, J. A., \& Blandón, L. C. (2016). La construcción de pueblo en el populismo. Análisis al discurso de un presidente en Colombia (2002 - 2010). Rev. Guillermo de Ockham, 14(2), 165-173. doi: http://dx.doi.org/10.21500/22563202.2753

\section{Resumen}

El objetivo de este artículo es presentar un análisis sobre las categorías que enmarcan la construcción de pueblo en el populismo, presentes en el discurso político del expresidente Álvaro Uribe Vélez. La metodología se fundamenta en la aplicación del método descriptivo multidimensional y en el análisis estadístico de datos textuales. Se trabajan 65 discursos emitidos por el líder político colombiano durante su período presidencial 2002-2010. Los resultados se soportan sobre la base de tres categorías propuestas por la teoría de la hegemonía como enfoque para el análisis político. La evidencia empírica referente de análisis son las palabras de mayor repetición que incentivaron la construcción de bandos dentro de la sociedad colombiana y la nominación de las políticas de gobierno desprendidas de esta relación. La pertinencia e innovación del estudio se fundamenta en su intensión de aportar nuevas herramientas metodológicas al populismo como fenómeno de análisis para la ciencia política y el conjunto de las disciplinas sociales en Colombia y América Latina.

Palabras clave: Populismo, hegemonía, discurso, presidente

\section{The construction of the town in populism: discourse analysis of a Colombian president (2002-2010)}

\section{Abstract}

The aim of this paper is to present an analysis of the categories that frame the construction the town in populism in the political discourse of former President Álvaro Uribe Vélez. The methodology is based on the application of multidimensional descriptive method and statistical analysis of textual data. 65 speeches issued by the Colombian political leader during his presidential period 2002-2010 were analyzed. The results are supported on the basis of three categories proposed by the theory of hegemony as an approach to political analysis. The empirical evidence concerning the analysis are the most repeated words which encouraged the construction of sides within Colombian society and the nomination of government policies that derive from this relationship. The relevance and innovation of the study is based on its intention to bring new methodological tools to populism as a phenomenon of analysis for political science and social studies set in Colombia and Latin America.

Keywords: Populism, hegemony, speech, president

\footnotetext{
* Artículo de Reflexión derivado del Seminario Investigativo "Enfoques para el Análisis Político I" orientado por Carlos A. Ramírez Ph.D. Programa de Ciencia Política, Universidad Javeriana, Cali-Colombia.

1. Profesional en Estudios Políticos y Resolución de Conflictos de La Universidad del Valle.

2. Profesional en Estudios Políticos y Resolución de Conflictos de La Universidad del Valle.
} 


\section{A construção de povo no populismo. Analisa 0 discurso de um presidente na Colômbia (2002-2010)}

\section{Resumo}

O objetivo deste artigo é apresentar um analise sobre as categorias que emolduram a construção de povo no populismo, presente no discurso político do ex-presidente Alvaro Uribe Velez. A metodologia baseia-se na aplicação do método descritivo multidimensional e análise estatística de dados textuais. Trabalhou 65 discursos emitidos pelo trabalho líder político colombiano durante o seu período presidencial 2002-2010. Os resultados encontram-se apoiados sobre a base de três categorias proposto pela teoria de hegemonia como uma abordagem para a análise político. A evidência empírica em matéria de análise são as palavras mais repeat encorajaram a construção de campos dentro da sociedade colombiana e da nomeação de políticas governamentais tirou essa relação. A relevância e inovação do estudo é baseado em sua intenção de fornecer novo populismo metodológico como um fenômeno da análise de ciência política e todas as disciplinas sociais em ferramentas de Colômbia e América Latina.

Palavras-chave: Populismo, hegemonia, discurso, presidente

\section{Introducción}

Una de las realidades indiscutibles de la política colombiana es la gran influencia del expresidente y hoy senador de la República Álvaro Uribe Vélez (AUV, en adelante), en su historia reciente. Este político ocupó durante ocho años la presidencia de Colombia tras una reforma constitucional (Acto Legislativo 02 del 2004) que aprobó su reelección inmediata. Posteriormente, gracias a su apoyo ancló a la cabeza del ejecutivo al hoy presidente Juan Manuel Santos, quien se posesionó en el cargo gracias a las banderas políticas impulsadas por este líder (especialmente en lo relativo a la seguridad). Hoy, el Senado de la República cuenta con su presencia y de la de diecinueve senadores que forman parte del Centro Democrático, partido creado, desarrollado y comandado por él.

Su popularidad como presidente fue una constante durante su gobierno. El promedio de favorabilidad en sus ocho ańos como fue del $72 \%$, cifra histórica comparada con la de sus antecesores. De hecho, cuando AUV se posesiona en el 2002 lo hace con un $69 \%$ de aprobación y se retira en el 2010 con un $75 \%$ (Londoño, 2010). Este fenómeno cobra trascendencia si se tienen en cuenta los numerosos escándalos y las polémicas generados durante su administración y en etapas posteriores.

En este orden de ideas, AUV se convierte en un personaje de sumo interés para cualquier estudioso de la política y de las ciencias sociales, pues factores como su extraordinaria capacidad de movilización de masas y la particularidad de su discurso, hacen que su estilo de gobierno se asocie con el concepto de populismo o el neopopulismo (Fierro, 2012). Es esta última construcción conceptual y teórica, la materia prima para configurar la siguiente pregunta de investigación orientadora del estudio: ¿̨uáles fueron las principales estrategias discursivas propias del populismo de las que se valió AUV para consolidar su propuesta como hegemónica durante su administración presidencial (2002-2010)?

En un primer paso se procede a la conformación del estado del arte con base en la literatura de vertientes académicas como la antropología, la sociología, la politología y la comunicación. Cárdenas (2012) hace un interesante recuento de los estudios de AUV como líder político y afirma que en términos de cantidad los estudios publicados en revistas científicas, tesis doctorales o de maestría son pocos si se comparan con los hechos a homólogos como Hugo Chávez.

Sin embargo, trabajos como los de Duque (2009) son importantes para un primer acercamiento en cuanto articulan el liderazgo de AUV como resultado de de tres categorías: 1 . el contexto; 2 . características y capacidades propias del líder; y 3. un vínculo de estas en relación con el pueblo. Destacan en este trabajo los rasgos conservadores y autoritarios de su gestión nominados por el autor. Por otro lado, se tiene la obra de Carrillo (2010), en la que analiza el discurso del hoy senador con base en insumos conceptuales de la teoría de Ernesto Laclau. Conceptos como significantes vacíos, hegemonía y retórica, entre otros, son articulados con el neopopulismo, este último definido, grosso modo, como el conjunto de características del populismo que tiende a concepciones de derecha y hacia el neoliberalismo. Finalmente, tenemos las ideas de Galindo (2006), quien se encarga de poner en duda la utilización tanto del concepto de populismo como el de neopopulismo -en el caso específico de AUV- y trae a colación aspectos ligados al contexto histórico del país 
marcado por una profunda fragmentación cultural y partidista que impide una formación sólida de "pueblo". Por ende, tras la utilización de las categorías de Kenneth Roberts concluye que no es adecuado situar a AUV dentro de los parámetros analíticos ofrecidos por las teorías del populismo. Estas discrepancias encontradas a lo largo de las investigaciones consultadas de estos y otros autores (Cárdenas, 2013, Rodríguez, 2013, Hoyos, 2010), evidencian un fenómeno en abierta discusión dispuesta a recibir aportes para el análisis desde cualquier frente de la ciencia social.

El marco teórico que servirá de soporte a la metodología y posterior discusión, se estructura sobre la base de los conceptos de populismo, discurso y hegemonía (Chomsky, 2015). El primero -el más amplio de los tres- es definido por Frei y Rovira (2008) como una impresión y una ambivalencia dada la carencia de consenso en la formación de un término genérico que sirva como marco de análisis para estudios de diverso corte sobre el tema. Aun así, Roberts (1999) mediante la nominación de características como liderazgos personalistas y procesos de movilización, acuña una relación de simpatía entre el líder y las masas, minimizando así el peso institucional e incrementando el contacto directo entre ambos actores, con lo cual pretende dar mayor cuerpo al concepto. Como valor agregado, un discurso polarizador de la sociedad y un proyecto económico en el que los sectores más deprimidos se ven recogidos, son elementos per se del populismo, según su clasificación.

Por otro lado, autores como Errejón (2011a, 2011b, 2012) y Laclau (2005) otorgan un peso trascendental al discurso como elemento polarizador de la sociedad, y profundizan en esta característica enlistada por Roberts. El discurso, en términos de Errejón, rebasa los campos de lo escrito y lo hablado. Más allá de lo evidente, el discurso y su eficacia en una relación entre pueblo y líder, envuelve un conjunto de entes externos que lo refuerza y lo constituye como la base de una dicotomía. Así, esta categoría se fija en prácticas e instituciones que construyen significado político merced a hechos determinados. Aspectos institucionales y culturales son esenciales para determinar el saber y el sentir populares. Es por esta razón que la fortaleza del discurso se mide en aquellos dispositivos que generen sentidos compartidos.

Lo que hay que buscar en cada caso no son solo las manifestaciones gráficas, escritas o verbales de un actor, sino el marco de significado desde el que la realidad social es procesada, explicada y ordenada para generar determinados posicionamientos (Errejón, 2011b, p.10).
Es precisamente de este recurso que se vale el líder y es uno de los aspectos fundamentales para pasar a una segunda categoría, a saber, la hegemonía (Carrillo, 2010). Laclau (2005), define la hegemonía como

la capacidad que tiene una diferencia sin dejar de ser particular, de asumir la representación de una totalidad inconmensurable. De esta manera, su cuerpo está dividido entre la particularidad que ella aún es y la significación más universal de la que es portadora (p. 95).

En este estado de cosas, la hegemonía es la materialización de un discurso polarizador que fragmenta la sociedad entre un grupo con aspiración de generalidad capaz de absorber las demandas de grupos dispares en una misma propuesta a través de la equivalencia, y grupos disidentes; es decir, la contrahegemonía. De esta manera, el populismo se vale de aparatos discursivos eficaces para agrupar a las masas en armonía con la propuesta del líder. Estas estrategias del discurso dividen la sociedad entre un pueblo soportado por la propuesta hegemónica y un bando disidente; es decir una relación amigo-enemigo (Mouffe, 2003; 2007). De esta manera, los sentidos compartidos cargados de significado político se determinan por la generación de un "nosotros" soportado en la existencia de un ellos (Mouffe, 1995, citada por Errejón, 2011b). De esta manera, la literatura propia de los enfoques para el análisis político que han estudiado el populismo y la hegemonía, conciben la política como un entorno de conflicto en el cual la lucha se configura por la construcción de los bandos. Es menester, por lo tanto, determinar como soporte teórico para responder la pregunta de investigación, las estrategias discursivas que consolidan la hegemonía. El enfoque constructivista propuesto por Errejón (2011), propone cuatro parámetros de relevancia intrínseca para el presente estudio:

1. Un "nosotros" que padece la problemática.

2. Un "ellos" responsable de la injusticia.

3. Una propuesta de solución imaginada como alcanzable.

4. Una motivación, referentes o aglutinantes simbólicos para la movilización en pos de los objetivos comunes del "nosotros".

Mediante esta operación, es posible comprender el modo como el discurso ordena el campo político colombiano y produce grupos, bandos y alineaciones en torno a una agenda determinada, todo ello con base en un proyecto político desarrollado durante ocho años en el gobierno de AUV. Así, sobre la base de las tres primeras categorías se analizará cómo en los discursos más relevantes se polariza 
la sociedad en virtud de un "ellos" y un "nosotros" y se hace una propuesta de solución a la problemática que aglutina los distintos grupos en la propuesta hegemónica.

\section{Método}

\section{Unidad de análisis}

La muestra fue seleccionada por el método de muestreo intencional u opinático (Lagares \& Puerto, 2001). Se tomó como criterio de selección la tipología de discursos políticos desarrollada por Gerstlé (2005,) que establece siete tipos de discursos políticos. Con base en dicho criterio, se seleccionaron seis discursos de balance, dieciocho discursos políticos de llamado, quince discursos didácticos, cuatro discursos polémicos, dos discursos de debate electoral, dieciséis de confirmación y cuatro de agregación. En total, se obtuvieron sesenta y cinco discursos políticos emitidos por AUV durante el período presidencial 20022010 tomados de su página web alvarouribevelez.com.co/ discursosmemorables (tablas 1 y 2).

Tabla 1

Total de la muestra

\begin{tabular}{lcc}
\hline \multicolumn{1}{c}{ Período presidencial } & Total & Porcentaje \\
\hline $2002-2006$ & 25 & 38,46 \\
\hline $2006-2010$ & 40 & 61,54 \\
\hline Total & 65 & 100 \\
\hline
\end{tabular}

Fuente: Elaboración propia.

Tabla 2

Total de discursos agrupados por tipos de discursos políticos (tipología de Gerstlé)

\begin{tabular}{lcc}
\hline \multicolumn{1}{c}{ Tipo de discurso político } & Total & Porcentaje \\
\hline De balance & 6 & 9,23 \\
\hline De llamado & 18 & 27,69 \\
\hline Didáctico & 15 & 23,08 \\
\hline Polémico & 4 & 6,15 \\
\hline De debate electoral & 2 & 3,08 \\
\hline De confirmación & 16 & 24,62 \\
\hline De agregación & 4 & 6,15 \\
\hline Total & 65 & 100 \\
\hline
\end{tabular}

Fuente: Elaboración propia.

\section{Procedimiento}

El ejercicio investigativo se estructuró en cuatro etapas:

1. Selección de la muestra de acuerdo con el criterio establecido (tipología de Gerstlé).

2. Construcción de una matriz en Excel que posibilitara el registro de los discursos (corpus) e undicara el periodo presidencial y el tipo de discurso político en el cual se inscribe cada unidad léxica (segmentos o palabras repetidas).

3. Identificación de las categorías desarrolladas por Errejón (2011b) a partir del análisis del contexto semántico de las palabras, con el fin de determinar la forma como se construye pueblo en AUV.

4. Análisis de los resultados con base en las herramientas teóricas de la hegemonía como enfoque para el análisis político.

La recolección de información se llevó a cabo durante quince días, desde el 10 de noviembre de 2014 hasta el 24 de noviembre de 2014.

\section{Diseño}

Desde la perspectiva metodológica de la investigación cuantitativa, esta exploración es definida por Peña (2000) citado en Becué, Lebart y Rajadell, (1992) como descriptiva multidimensional, en cuanto se centra en la aplicación de análisis de correspondencia con el fin de determinar la asociación de palabras en los discursos políticos analizados. Por su parte, el análisis de los datos se hace a partir del análisis estadístico de datos textuales (AEDT), centrado únicamente en el nivel de análisis unidimensional.

Dado que la unidad de análisis es textual (palabras repetidas), según Torruella y Llisterri (1999, citados por Sánchez, L., López, W., \& Barreto, 2013), el tipo de diseño adoptado en este ejercicio investigativo corresponde al corpus especializado que acopia textos en un periodo determinado.

\section{Umbrales de frecuencia elegidos}

Con el fin de establecer los segmentos o palabras que formarían parte del análisis unidimensional, se estableció un umbral de 20; por tanto, se trabajó con aquellas palabras cuya frecuencia fuera igual o mayor a 21 .

\section{Resultados}

Los resultados que se presentan en este apartado se organizan en tres categorías identificadas en los discursos políticos de AUV de la siguiente manera: "nosotros", "ellos" y "una propuesta de solución".

En la categoría "nosotros", definida por la existencia de un "ellos", se ubican aquellos segmentos o palabras relacionados con la delimitación de un sujeto colectivo de problemas y expectativas compartidas por el líder político AUV (Errejón, 2011b). En la categoría "ellos" se incluyen palabras o segmentos que hacen referencia a grupos de la sociedad colombiana identificados como antagonistas o 
adversarios, que generan ordenamientos contrapuestos de las fidelidades y la identidad política de AUV (Anderson, citado por Errejón, 2011b).

Finalmente, la categoría de "una propuesta de solución" incluye las palabras o segmentos repetidos en los diversos discursos emitidos por AUV que describen o refieren la solución política alcanzable para el problema de las injusticias que padece Colombia.

\section{Categoría nosotros}

La palabra identificada con más frecuencia en los discursos políticos de AUV en el marco de la categoría "nosotros" es patria. ${ }^{3}$ Adicionalmente, palabras como pueblo y compatriotas, directamente asociadas al sentimiento de identidad que vincula al individuo con su patria, también presenta una significativa frecuencia en los discursos políticos (Tabla 3).

Tabla 3

Palabras repetidas en la categoría "nosotros"

\begin{tabular}{lc}
\hline \multicolumn{1}{c}{ Vocabulario de palabras “nosotros” } \\
\hline Palabra & Frecuencia \\
\hline Pueblo & 288 \\
\hline Patria & 326 \\
\hline Compatriotas & 280 \\
\hline Amigos & 34 \\
\hline
\end{tabular}

Fuente: Elaboración propia.

En relación con los segmentos repetidos, se encuentra que "la comunidad" es el segmento de mayor frecuencia en los discursos políticos analizados. Por su parte, los segmentos "mis compañeros" y "los ciudadanos" acompañan con menor frecuencia el discurso político en esta categoría. Respecto de estos últimos segmentos, es importante señalar que el primero se centra en la concepción de la identidad política construida y compartida por el líder AUV, a saber, la seguridad democrática; el segundo hace referencia al carácter de legalidad -por cuanto excluye a los grupos armados al margen de la ley (ellos)- de quienes residen en la comunidad política y a quienes va orientado su discurso y dirigidas sus políticas (Tabla 4).

Tabla 4

Segmentos repetidos en la categoría "nosotros"

\begin{tabular}{lc}
\hline \multicolumn{2}{c}{ Segmentos “nosotros” } \\
\hline \multicolumn{1}{c}{ Segmentos } & Frecuencia \\
\hline Mis compañeros & 29 \\
\hline Los ciudadanos & 40 \\
\hline La comunidad & 239 \\
\hline Fuente: Elaboración propia.
\end{tabular}

Fuente: Elaboración propia.

\section{Categoría ellos}

En primer lugar, se observa que la palabra con mayor frecuencia es "terrorismo", la cual delimita el contexto sobre el cual es interpretado el adversario o antagonista. En relación a esta palabra, se desprenden otras como "terroristas", "cobardes", "criminales", que0 operan como connotaciones negativas de la categoría "ellos". Luego, palabras como FARC e izquierda son encontradas en los discursos políticos como alusión, en el primer caso, a un grupo al margen de la ley que no comparte la identidad política dominante y, en el segundo caso, a un sector legal de la sociedad que se diferencia del "nosotros" a partir de la generación de ordenamientos contrapuestos al del líder AUV (Tabla 5).

Tabla 5

Palabras repetidas en la categoría "ellos".

\begin{tabular}{lc}
\hline \multicolumn{1}{c}{ Palabra } & Frecuencia \\
\hline Terrorismo & 443 \\
\hline Criminales & 93 \\
\hline Izquierda & 82 \\
\hline Cobardes & 21 \\
\hline FARC & 195 \\
\hline Terroristas & 216 \\
\hline Fuente: Elaboración propia. &
\end{tabular}

El segmento con la frecuencia más alta es "enemigos del gobierno", el cual en el discurso hace referencia a aquellos ciudadanos, grupos al margen de la ley, partidos y movimientos políticos y, en general, personas que no comparten las tesis políticas defendidas por el gobierno de AUV. Junto a este segmento aparece "esos bandidos" que al igual que en el caso de las palabras "criminales", "cobardes" y "terroristas", opera como adjetivo calificativo de "ellos" (Tabla 6).

Tabla 6

Segmentos repetidos en la categoría "ellos"

\begin{tabular}{lc}
\hline \multicolumn{1}{c}{ Segmentos } & Frecuencia \\
\hline Enemigos del gobierno & 22 \\
\hline Esos bandidos & 21 \\
\hline Fuente: Elaboración propia. &
\end{tabular}

\section{Una propuesta de solución}

Las palabras con mayor número de repeticiones en esta categoría están referidas a los valores democráticos que sustentan la propuesta política de AUV. Así, por ejemplo, palabras como "seguridad", "democracia", "justicia" y "paz", son mencionadas en sus discursos como

3. Se usa las cursivas para señalar aquellas expresiones que fueron directamente identificadas en los discursos políticos analizados. 
valores realizables a través de la política de Seguridad Democrática. De igual manera, se identifican palabras que si bien no son concebidas en el discurso como parte de la solución, se perfilan como la consecuencia de llevar a cabo la política de seguridad concebida por el gobierno de AUV como a solución a los problemas e injusticias de la sociedad colombiana. Estas son "prosperidad" y "equidad", elementos constitutivos de una comunidad política deseable (Tabla 7).

Tabla 7

Palabras repetidas en la categoría "una propuesta de solución"

\begin{tabular}{lc}
\hline \multicolumn{2}{c}{ Palabras de "una propuesta de solución" } \\
\hline Seguridad & Frecuencia \\
\hline Democracia & 545 \\
\hline Justicia & 369 \\
\hline Prosperidad & 308 \\
\hline Equidad & 62 \\
\hline Paz & 89 \\
\hline
\end{tabular}

Fuente: Elaboración propia.

En primer lugar, es claro que el segmento con mayor frecuencia es "seguridad democrática", relacionado directamente con la política de seguridad diseńada y ejecutada por AUV a lo largo de su periodo presidencial (2002-2010). En relación con este segmento, se encuentran "lucha frontal" y "fuerza pública", ambos concebidos como estrategias del gobierno para derrotar a los grupos armados al margen de la ley. Este último, objetivo esencial de la propuesta política de seguridad.

Por otro lado, el segmento "cohesión social", aparece (junto a la seguridad democrática y la confianza inversionista) como uno de los tres pilares del accionar del gobierno de AUV. En los discursos políticos se entienden como la fuente de la "perdurabilidad democrática" (Tabla 8).

Tabla 8

Segmentos repetidos de "una propuesta de solución"

\begin{tabular}{lc}
\hline \multicolumn{2}{c}{ Segmentos de "una propuesta de solución” } \\
\hline Segmento & Frecuencia \\
\hline Seguridad democrática & 294 \\
\hline Cohesión social & 104 \\
\hline Fuerza pública & 111 \\
\hline Lucha frontal & 32 \\
\hline
\end{tabular}

Fuente: Elaboración propia.

A continuación, para sintetizar de manera gráfica la frecuencia de las palabras y los segmentos descritos en las tres categorías de análisis, se situará en el cuerpo del apartado un diagrama de dispersión (Figura 1) que presenta de manera conjunta todas las palabras objeto de análisis.
El gráfico consta de un eje principal $(\mathrm{Y})$ en el cual se ubican de manera ordenada cada palabra y segmento en un intervalo de 21 a 600. el eje $\mathrm{X}$ no posee cifras, pues no es necesario para mostrar la repetición de la frecuencia de las palabras. Por esta razón, este eje es el espacio de cada uno de los términos en relación con cada repetición.

\section{Figura 1}

Diagrama de dispersión de frecuencia de palabras y segmentos

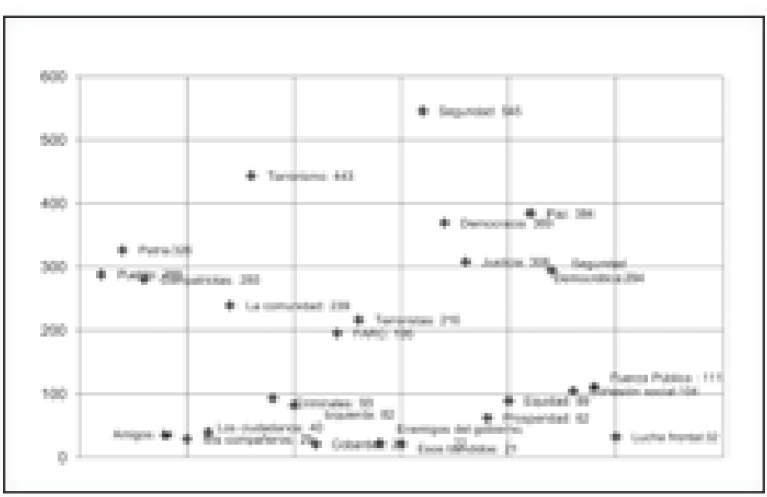

Fuente: Elaboración propia.

\section{Componentes característicos del discurso político de AUV según la tipología de Gerstlé}

Las expresiones que caracterizan al discurso político de balance se ubican en la categoría "ellos". En efecto, palabras como "FARC", "terrorismo" y "terrorista" ocupan el 45,5\% de las palabras repetidas en los discursos de balance. Sin embargo, junto a esta categoría aparece, en segundo lugar con un 32,9\%, el uso de palabras como "seguridad" y "lucha" correspondientes a la categoría "solución". La palabra más relacionada en este tipo de discursos es "seguridad" con 23 repeticiones, la cual fue expuesta en el vocabulario de palabras como valor democrático supremo.

Por su parte, el discurso político de llamado se soportó mayoritariamente $(70,7 \%$, respecto del uso y repetición de palabras inscritas en otras categorías) sobre la categoría "solución" con la utilización reiterada de las palabras "seguridad", "democracia" y "paz", esta última la palabra más referenciada por AUV en estos discursos (noventa y cinco repeticiones).

$\mathrm{Al}$ igual que en el caso anterior, los discursos políticos didácticos fueron elaborados por el líder con base en la categoría "solución" (84,7\%). Sin embargo, a diferencia de los discursos de llamado, aquí la palabra con mayor frecuencia es "seguridad", con 140 menciones.

Las expresiones que distinguen el discurso polémico se ubican en la categoría "solución". Por lo anterior, a diferencia de unas pocas menciones (dos) que se hace de 
palabras como "FARC" y "terroristas" (categoría "ellos"), no hay palabras relacionadas con "nosotros" y "ellos", usadas exclusivamente en este tipo de discurso. Por ende, la categoría "solución" constituye el 71,4\% del total de categorías usadas por AUV en los discursos polémicos, y es "democracia", con cinco repeticiones, la palabra con mayor frecuencia.

Asimismo, los discursos políticos de debate electoral se estructuran a partir del uso de palabras como "seguridad" y "democracia", enmarcadas en la categoría "ellos". Sin embargo esta categoría constituye el 48,5\% de las categorías presentes en los discursos de este corte. Ahora bien, sobre la base de que no pertenece a la categoría dominante la palabra con mayor frecuencia es "FARC" (ellos) con catorce menciones.

En relación con los discursos políticos de confirmación, las expresiones que caracterizan este discurso se agrupan en las categorías "nosotros" y "solución", con un 31,67 \% y $47,9 \%$, respectivamente. Respecto de las palabras o segmentos con mayor frecuencia, la palabra paz se ubica con 98 repeticiones.

Finalmente, los discursos políticos de agregación se caracterizan, al igual que la mayor parte de los anteriores discursos analizados, por el uso de palabras que se agrupan bajo la categoría "solución" (67,5\%). Así, "democracia", "equidad" y "seguridad democrática” son las palabras con mayor frecuencia en los discursos de agregación emitidos por AUV.

\section{Discusión}

Las palabras, figuras, símbolos y objetos adoptados por propuestas con aspiraciones hegemónicas, desempeñan un papel fundamental en los estilos populistas, pues se encargan de configurar significantes vacíos que incentivan la construcción de un pueblo que se autoproclama como la totalidad (Duque, 2013). Cada uno de estos mecanismos discursivos se caracteriza por poseer un carácter amplio de generalidad, hasta el punto de agrupar sectores heterogéneos en una misma propuesta. Carrillo (2010) afirma que estos términos son tan poco específicos y su significado tan vacío que se podrían generar equivalencias en las cuales muchos grupos, por disímiles que sean, no se sienten excluidos de la propuesta totalizadora. Es así como esta categoría abordada por Laclau (2005) entendida como "palabras-imágenes" o "términos privilegiados" es definitiva para él, toda vez que configuran la condición misma de la hegemonía (Laclau, 1996 citado por Montero 2013).
Este concepto toma, entonces, trascendencia para la presente discusión, pues cada palabra y segmento contabilizados fueron una constante en los sesenta y cinco discursos analizados. Sin importar el tipo (balance, didáctico, polémico, etc.), la coyuntura política o el contexto en el que fueron pronunciados, determinados términos se utilizaban de manera constante y cotidiana por el expresidente AUV y en ellos se evidencia la intención de polarizar la sociedad entre un "ellos" y un "nosotros", además de la puesta en escena de una solución a la problemática vivida por el "nosotros" y generada por el "ellos", provocando así una relación víctima-victimario, amigo-enemigo.

Dos de los términos que mayor respaldo proveen a la anterior afirmación fueron "terrorismo" y "terrorista". Estas palabras tuvieron una gran cantidad de repeticiones (216 y 443, respectivamente) en los 65 discursos objeto de estudio. Estas construcciones discursivas son significantes vacíos con una fuerte carga de connotación negativa que al asociarse con palabras como "FARC", "bandidos", "criminales" e "izquierda" generaron la conjunción, en un grupo homogéneo, de un enemigo común disímil al pueblo. En los múltiples discursos y en contextos como posesiones presidenciales (dos, en el caso de AUV), homenajes y reconocimientos, toma de posiciones en momentos coyunturales de orden nacional e internacional y en la rendición de cuentas de resultados de la gestión administrativa, se evidenciaba la presencia de los términos nominados como causantes de las problemáticas económicas, sociales y políticas del país. De este modo, AUV en ocho años de administración polarizó al país entre el terrorismo y las victimas del terrorismo.

En este orden de ideas y sin tener en cuenta las implicaciones y repercusiones que genera para alguien ser acusado de terrorista (Casas, Ramírez \& Caro, 2011), se evidenció la utilización de manera sistemática de este recurso no solo para referirse a las FARC grupo insurgente con el cual se asoció el término en la mayoría de los discursos seleccionados (además de los otros juicios de valor nominados), sino también para movimientos sociales, ONG, periodistas o simplemente disidentes de las propuestas de gobierno (Flórez, 2011).

Por otro lado, tuvo gran protagonismo el establecimiento del pueblo mediante un conjunto estratégico de significantes vacíos representados en el estudio por palabras y segmentos adoptados y puestos en escena, según las demandas del contexto. Términos como "pueblo", "compatriotas", "amigos", "mis compañeros", "patria", "ciudadanos" y "comunidad" fueron una amalgama de insumos propuestos por AUV para articular en una misma 
línea discursiva sus políticas de gobierno y su propósito de adherir las masas a su propuesta. Por esta razón, un denominador común en el análisis de su discurso fue la utilización en un mismo espacio de estos términos integradores junto con juicios de valor y palabras de connotación negativa, evidenciándose así la intención de dividir y construir un pueblo en el conjunto de la población colombiana.

Como valor agregado, las palabras y segmentos aglomerados en la categoría tres son resultado de una polarización sobre la cual el líder cimentó sus políticas de gobierno. Por esta razón, expresiones como "cohesión social" y "seguridad democrática" tuvieron alta repetición en los discursos estudiados, pues eran la brújula que orientaba el plan de desarrollo en las dos administraciones presidenciales. Así, con el análisis de varios tipos de discurso se evidencia que AUV es un político consecuente a la hora de polarizar la sociedad e introducir en contextos en los cuales se expresa, un conjunto repetido de términos y estrategias discursivas que lo articulan al conjunto de la sociedad en virtud de la victimización generada por un bando enemigo, razón por la cual es necesario implementar políticas públicas en las que el enemigo esté implicado por ser el causante principal de las problemáticas comunes.

El presente estudio permite, entonces, aproximarse a tres conjeturas generales. La primera, es que a pesar de la dificultad de encasillar el estilo de gobierno de AUV dentro del populismo o el neopopulismo, dada la carencia de consenso académico respecto del uso de estos conceptos y de los estudios que se han aproximado a este fenómeno, es posible destacar los rasgos populistas de este mandatario en su discurso político, pues como se evidenció, su discurso, a pesar de la diversidad de contextos y situaciones en los que es pronunciado, posee unos parámetros comunes en cuanto al uso de estrategias discursivas que se constituyen en rasgos populistas. En segundo lugar destaca la importancia de los estudios sobre hegemonía como enfoque para el análisis político. En este sentido, Errejón (2011) hace una crítica hacia "la ciencia política dura", sistematizadora de datos institucionales, jurídicos o electores, y propone que la disciplina centre su atención en fenómenos reales, aunque en ocasiones difusos, escurridizos y difíciles de analizar mediante el rigor metodológico impuesto por los paradigmas de la investigación social. Es así como el presente estudio trató de combinar ambas posturas: por un lado, se enfocó en el populismo y la hegemonía como fenómenos de análisis; y por el otro, se adoptó como parámetro metodológico para el análisis una herramienta estadística configurada como maniobra de repetido uso en el análisis electoral, campo intrínseco de la politología.
Por último, es menester situar como tópico importante la discusión -citada a manera de epígrafe en el presente estudio- hacia la que nos lleva Laclau (2005); es decir, la construcción de pueblo como condición sine qua non para la democracia que, en efecto, es desarrollada por el populismo como se hizo manifiesto el presente estudio. El populismo puede situarse en distintos contextos como una antinomia, pues por un lado genera un pueblo politizado, apropiado y conocedor de sus necesidades, que ve en el Estado un que garantiza el cumplimiento de derechos civiles, económicos y políticos; pero por por el otro, pierde utilidad al hacer de la opinión pública un elemento favorable para que el líder tome decisiones alejadas del bien común. Sin embargo, a pesar de esta ambivalencia y del carácter peyorativo que pueda llegar a tener, el populismo puede ser un fenómeno sumamente eficaz para darle cuerpo al abstracto concepto de pueblo, y es, sin duda, un asunto que suscita un largo debate académico sobre la realidad que atraviesan los países latinoamericanos en la actualidad.

\section{Referencias}

Becué, M., Lebart, L., \& Rajadell, N. (1992). El análisis estadístico de datos textuales. La lectura según los escolares de enseñanza primaria. Anuario de psicología, 55, 7-22.

Sánchez, L., López, W., \& Barreto I (2013). Enmarcamiento cognitivo de la cultura política. Un análisis desde las redes sociales en internet (Twitter). Revista interamericana de psicología, 3, 383-394.

Cárdenas, F. (2012). Aparato discursivo del expresidente Álvaro Uribe Vélez. Horizontes miméticos del pensamiento hegemónico neoliberal en Colombia (2002 - 2010). Análisis politico, 25(75), 139-157.

Cárdenas, F. (2013). (Des) orden y signos políticos dominantes del expresidente colombiano Álvaro Uribe Vélez. Estudios Politicos, 42, 85-111.

Carrillo, C. (2010). Análisis del discurso de Álvaro Uribe Vélez (2002-2006) bajo una lógica neo populista. (Monografía presentada para optar al título de politóloga). Bogotá, Universidad del Rosario.

Casas, Z., Ramírez, M., \& Caro, A. (2011). La oscuridad que encierra el término terrorista: una cuestión aún por definir. Revista semilleros de Investigación, 3, 115-119.

Congreso de la República de Colombia. Acto legislativo 02 de 2004. (2004). Por el cual se reforman algunos artículos de la Constitución Politica de Colombia y se dictan otras disposiciones. Recuperado de https://goo.gl/OrKsO7

Chomsky, N. (2015). Impacts of free market and US foreign policy on Colombian and Latin American revolution. Rev. Guillermo de Ockham, 13(1), 21-25.

$172 \varangle$ Universidad de San Buenaventura, Cali - Colombia 
Duque, G. (2013). El populismo abortado: los significantes vacíos en el gobierno del General Gustavo de Gustavo Rojas Pinilla. Trans-pasando Fronteras, 4, 67-91.

Duque, J. (2009) Álvaro Uribe Vélez: Populismo, autoritarismo y conservadurismo. En J. Duque (Ed.), Liderazgo politico en sociedades modernas. (pp. 15-32). México: Colegio de Veracruz.

Errejón, I. (2011a). La construcción discursiva de identidades populares. Viento sur, 114, 75-85.

Errejón, I. (2011b). ¿Qué es el análisis político? Una propuesta desde la teoría del análisis del discurso y la hegemonía. RELACSO, 1(4), 1-16.

Errejón, I. (2012). La lucha por la hegemonía en Bolivia (2006 - 2009). Un análisis discursivo. (Tesis de doctorado). Universidad Complutense de Madrid, España.

Fierro, M. (2012). Álvaro Uribe Vélez: Neopopulismo, retórica y asimilación del discurso por los bogotanos. (Tesis de maestría). Universidad Nacional de Colombia, Bogotá.

Flórez, F. (2011). Álvaro Uribe y el terrorismo. Recuperado de https://goo.gl/tE6mEZ

Frei R., \& Rovira C. (2008). El populismo como experimento político: historia y teoría política de una ambivalencia. Revista de sociología, 22, 115-144.

Galindo, C. (2007). Neopopulismo en Colombia: El caso de Álvaro Uribe Vélez. Iconos. Revista de Ciencias Sociales, 27, $147-162$
Gerstlé, J. (2005). La comunicación Política. Editorial LOM. Chile.

Hoyos, G. (2010). Estado de opinión: ¿información, comunicación y lenguaje público? Signo y pensamiento, Vol 29. Bogotá, Colombia.

Laclau, E. (2005). La razón populista. Buenos Aires: Fondo de cultura económica.

Lagares, P. \& Puerto, J. (2001). Población y muestra. Recuperado de https://goo.gl/ju8M0A

Londoño, J. (2010). La de Uribe, una histórica popularidad. Recuperado de https://goo.gl/8iefXB.

Montero, A. (2012). Significantes vacíos y disputas por el sentido en el discurso político: un enfoque argumentativo. Identidades, 3, 1-25.

Mouffe, Ch. (2007). En torno a lo político. Buenos Aires: Fondo de Cultura Económica.

Mouffe, Ch. (2003). La paradoja democrática. Barcelona: Gedisa.

Roberts, K. (1999). El neoliberalismo y la transformación del populismo en América Latina: el caso peruano. En. K. Roberts (Ed.), Populismo y neopopulismo en América latina. El caso de la cenicienta. (pp.35-48). Buenos Aires: Eudeba.

Rodríguez, A. (2013). El Estado de opinión: La construcción del discurso de Seguridad en el gobierno de Álvaro Uribe Vélez. (Tesis de maestría). Facultad Latinoamericana de Ciencias Sociales, Ecuador. 